\title{
Subgroups generated by images of endomorphisms of Abelian groups and duality
}

\author{
Grigore Călugăreanu, Andrey R. Chekhlov and Piotr A. Krylov
}

Communicated by Pavel A. Zalesskii

\begin{abstract}
A subgroup $H$ of a group $G$ is called endo-generated if it is generated by endoimages, i.e. images of endomorphisms of $G$. In this paper we determine the following classes of Abelian groups: (a) the endo-groups, i.e. the groups all of whose subgroups are endo-generated; (b) the endo-image simple groups, i.e. the groups such that no proper subgroup is an endo-image; (c) the pure-image simple, i.e. the groups such that no proper pure subgroup is an endo-image; (d) the groups all of whose endo-images are pure subgroups; (e) the ker-gen groups, i.e. the groups all of whose kernels are endo-generated. Some dual notions are also determined.
\end{abstract}

\section{Introduction}

In what follows we use the short term endo-image for an endomorphic image of a group.

As early as 1955, Fuchs, Kertesz and Szele determined (see [7]) the Abelian groups all of whose subgroups are endo-images denoting by $P$ this property. A torsion group has this property iff all its primary components have this property.

Their results are the following.

Theorem. A p-group $G$ has property $P$ iff the final rank of $G$ is equal to that of a basic subgroup $B$ of $G$ iff $G$ is a homomorphic image of $B$.

Theorem. A group $G$ with torsion-free rank $r=r_{0}(G)>0$ has property $P$ iff

(i) in the case $r<\boldsymbol{\aleph}_{0}$, the group $G$ is of the form $T \oplus \bigoplus_{r} \mathbb{Z}$, where $T$ is a torsion group with property $P$ (covered by the previous theorem),

(ii) in the case $r \geq \boldsymbol{\aleph}_{0}$, the group contains a free direct summand of rank $r$ and in the torsion subgroup $T(G)$,

each primary component $G_{p}$ of final rank $>r$ is a p-group with property $P$.

Corollary. A torsion-free group $G$ has property $P$ iff $G$ is either a free group of finite rank or has a free direct summand of rank $|G|$. 
In the same paper the authors notice that for a group $G$ all subgroups are endoimages iff all subgroups that are direct sums of cyclic groups are endo-images.

In particular, any bounded group has property $P$ and a countable p-group fails to have this property iff it has a non-zero divisible part (i.e. direct summands $\left.\mathbb{Z}\left(p^{\infty}\right)\right)$ and its reduced part is bounded.

In the sequel, the word "group" means always "Abelian group". For a group $G$, $E=: \operatorname{End}(G)$ denotes the endomorphism ring. For a subset $X$ of a torsion-free group $G,\langle X\rangle_{*}$ denotes the pure subgroup generated by $X$ (i.e. the intersection of all pure subgroups which include $X$ ). For other unexplained notions and notation we refer to [5] and [6].

It is natural to consider the following generalization:

Definition. Let $H$ be a subgroup of a group $G$ and let $E=$ : $\operatorname{End}(G)$. We say that $H$ is endo-generated if $H=\sum\{f(G): f \in E, f(G) \leq H\}$. Obviously, every endo-image of $G$ is endo-generated. In particular, as images of idempotent endomorphisms, direct summands are endo-images and so endo-generated. Further, a group all of whose subgroups are endo-generated will be called an endo-group.

However, since every endomorphism $f \in E$ such that $f(G) \leq H$ determines (and is determined by) a homomorphism $f: G \rightarrow H$, notice that the above notions may be equivalently defined using a more general well-known construction: for groups $A$ and $B$ set $S_{A}(B):=\sum_{\alpha \in \operatorname{Hom}(A, B)} \alpha(A)$, a fully invariant subgroup of $B$ called the $A$-socle of $B$ (or the trace of $A$ in $B$ ). If $S_{A}(B)=B$, we say that $B$ is $A$-generated. Moreover, the group $B$ is called finitely $A$-generated if there exist finitely many homomorphisms $\varphi_{i}: A \rightarrow B, i=1, \ldots, n$, such that $B=\sum_{i=1}^{n} \varphi_{i}(A)$.

Therefore, a subgroup $H$ of a group $G$ is (finitely) endo-generated iff $H$ is (finitely) $G$-generated and a group $G$ is a (finite) endo-group iff all its subgroups are (finitely) $G$-generated.

The plan of the paper is the following: in Section 2, the endo-groups are completely determined and in Section 3 we determine the endo-image simple groups (i.e. the groups $G$ in which the only endo-images are 0 and $G$ ) and then we determine two other classes of groups introducing pure subgroups into our discussion: no pure subgroup is an endo-image, or, all endo-images are pure subgroups.

In Section 4 we determine for groups a notion introduced in [12]: ker-gen groups, that is, groups that generate their kernels (i.e. for every $f \in E, \operatorname{ker}(f)$ is endo-generated).

In Section 5, we study some dual notions to those mentioned above. The paper ends with a list of open problems. 


\section{Endo-groups}

Examples. All groups with property P are endo-groups (i.e. all endo-images are endo-generated).

Since all subgroups of $\mathbb{Z}$ are endo-images (of the multiplications), $\mathbb{Z}$ is more than an endo-group. So are all simple groups, i.e. $\mathbb{Z}(p)$ for any prime $p$. Moreover, all cyclic groups are endo-groups and elementary groups are endo-groups.

Note that $\mathbb{Q}$ is not an endo-group. Indeed, all proper (which properly include $\mathbb{Z}$ ) subgroups (i.e. the rational groups) are not endo-generated. Actually more holds: $\mathbb{Q}$ has no proper endo-images and the same is true for $\mathbb{Z}\left(p^{\infty}\right)$.

Alternatively (Szele), $\operatorname{End}(G)$ is a field iff $G$ is isomorphic to $\mathbb{Q}$ or $\mathbb{Z}(p)$ for some prime $p$. These have no proper endo-images.

It is easy to check that if $H$ is a fully invariant subgroup of an endo-group $G$, then $G / H$ is also an endo-group.

First notice that $G$-generated subgroups abound. Indeed, we can prove the following:

Proposition 1. (1) In any separable group $G$ all pure fully invariant subgroups are G-generated.

(2) In any group $G$ all torsion pure subgroups are $G$-generated.

Proof. (1) Let $0 \neq x \in F$, where $F$ is a pure fully invariant subgroup of $G$. Since $G$ is separable, we have $x \in G_{1} \oplus \cdots \oplus G_{n}$, where $r\left(G_{i}\right)=1$ and $i=1, \ldots, n$. Then $F^{\prime}=F \cap\left(G_{1} \oplus \cdots \oplus G_{n}\right)=\left(F \cap G_{1}\right) \oplus \cdots \oplus\left(F \cap G_{n}\right)$ and $F \cap G_{i}=G_{i}$ if $F \cap G_{i} \neq 0$. Consequently, $F^{\prime}$ is a direct summand of $G$ and since $x \in F^{\prime}, F$ is $G$-generated.

(2) Let $H$ be a torsion pure subgroup of $G$. Then $H=D \oplus K$, where $D$ is the divisible part of $H$ and $K$ is reduced. Since $H$ is pure in $G$, so is $K$ and so $K$ is included in some reduced subgroup of $G$. If $D \neq 0$, then $D$ is a direct summand of $G$ so $D$ is $G$-generated. The subgroup $K$ is also $G$-generated since $K$ is generated by cyclic direct summands of $G$.

Some more examples are given in the following

Proposition 2. (1) In any homogeneous torsion-free separable group $G$, any pure subgroup is $G$-generated.

(2) Let $G=\prod_{i \in I} G_{i}$, where $G_{i}$ are torsion-free groups of rank 1 and same type and $I$ is infinite. Then every pure subgroup of $G$ is $G$-generated iff the type of each $G_{i}$ is idempotent. 
(3) Let $G=\prod_{i \in I} G_{i}$ be a vector group, where $G_{i}$ are torsion-free groups of rank 1 and type $\mathbf{t}_{i}=\mathbf{t}\left(G_{i}\right)$. Then every pure subgroup of $G$ is $G$-generated iff for any $0 \neq x \in G$ there exists a type $\mathbf{t}_{i}$ such that $\mathbf{t}_{i} \leq \mathbf{t}(x)$.

Proof. (1) According to [6, Proposition 87.2], any pure subgroup of finite rank is a direct summand of $G$. Whence the conclusion.

(2) If the set $I$ is infinite and the type $\mathbf{t}=\mathbf{t}\left(G_{i}\right)$ is not idempotent, then $G$ has a pure subgroup $H$ of rank 1 and $\mathbf{t}(H)<\mathbf{t}$. Then from [6, Lemma 96.1] it follows that $\operatorname{Hom}(G, H)=0$, i.e. $H$ is not $G$-generated. Conversely, according to [6, Lemma 96.4] the group $G$ is separable and homogeneous, and we use (1).

(3) Let $H=\langle x\rangle_{*}$. Since $\operatorname{Hom}(G, H) \neq 0$, by [6, Lemma 96.1], $\mathbf{t}_{i} \leq \mathbf{t}(x)$ for some $\mathbf{t}_{i}$. Conversely, let $F$ be a pure subgroup of $G$ and $0 \neq x \in F$. Since $\mathbf{t}_{i} \leq \mathbf{t}(x)$, the corresponding group $G_{i}$ homomorphic generates $\langle x\rangle_{*}$. Since every homomorphism $G_{i} \rightarrow\langle x\rangle_{*}$ lifts to some endomorphism of group $G$, it follows that $F$ is $G$-generated.

In what follows, we completely determine the endo-groups (i.e. the groups all of whose subgroups are endo-generated), a class of groups, larger than the class defined by property $\mathrm{P}$ (the groups all of whose subgroups are endo-images), in the Introduction.

We mention that, under the name of self-generators, endo-groups were characterized in [9, Theorem 2.1], using equivalences of module categories. For the sake of completeness, we have provided a direct (specific) proof below.

We already noticed that the definition of an endo-generated subgroup of a group $G$ and the definition of a $G$-generated subgroup are equivalent.

Let $A_{i}, B(i \in I)$ be groups and let $f_{i}: A_{i} \rightarrow B(i \in I)$ be homomorphisms. If $f: \bigoplus_{i \in I} A_{i} \rightarrow B$ is the factorization homomorphism, it is well known that $f\left(\bigoplus_{i \in I} A_{i}\right)=\sum_{i \in I} f_{i}\left(A_{i}\right)$. Hence $S_{A}(B)=B$ (i.e. $B$ is $A$-generated) iff $B$ is an epimorphic image of some $A$-free group (i.e. an arbitrary direct sum of copies of $A$ ), and a group is finitely $A$-generated iff an epimorphism $\bigoplus_{n} A \rightarrow B$ exists for some positive integer $n$.

Since every cyclic group is a homomorphic image of the group $\mathbb{Z}$, we immediately obtain the following:

Lemma 3. If the group $G$ has a direct summand isomorphic to $\mathbb{Z}$, then $G$ is an endo-group.

For torsion-free or mixed groups the converse also holds.

Proposition 4. Let $G$ be a torsion-free group or a (genuine) mixed group. Then $G$ is an endo-group iff $G$ has a direct summand isomorphic to $\mathbb{Z}$. 
Proof. Since one way is already covered by the previous lemma, suppose $G$ is an endo-group and let $H$ be a subgroup of $G$ with $H \cong \mathbb{Z}$. Since $H$ is $G$-generated, $H$ is an epimorphic image of some $G$-free group and so a non-zero homomorphism $f: G \rightarrow H$ exists with $\operatorname{Im} f \cong \mathbb{Z}$. Hence $G=A \oplus \operatorname{ker} f$, where $A \cong \operatorname{Im} f$, as desired.

As for torsion groups, it suffices to consider $p$-groups. Note that if $C \cong \mathbb{Z}(n)$, then $S_{C}(G)=G[n]$ for any group $G$.

Proposition 5. A p-group $G$ is an endo-group iff its reduced part is unbounded or $G$ is a bounded group.

Proof. To show that the conditions are necessary, assume the opposite, that is $G=B \oplus D$, where $0 \neq D$ is a divisible group and $p^{k} B=0$. The group $D$ is isomorphic to a direct sum of groups $\mathbb{Z}\left(p^{\infty}\right)$. Since all non-zero endomorphisms of $\mathbb{Z}\left(p^{\infty}\right)$ are epimorphisms, it follows that the subgroups $G\left[p^{m}\right]$ for any $m>k$ are not $G$-generated.

Conversely, let $G=A \oplus D$, where $A$ is an unbounded group, $D$ is a divisible group or $D=0$ and let $H$ be a subgroup of $G$. For any $k \geq 1$, a decomposition $A=C \oplus B$ exists, where $C \cong \mathbb{Z}\left(p^{n}\right)$ and $n \geq k$. Then clearly $S_{G}(H) \supseteq H\left[p^{k}\right]$ and so $S_{G}(H)=H$.

In the remaining case, let $G$ be a bounded group and $0=p^{k+1} G \neq p^{k} G$. Then $S_{\mathbb{Z}\left(p^{k}\right)}(H)=H$, whence $S_{G}(H)=H$, so $H$ is $G$-generated.

Since the structure of endo-groups is determined, we can easily deduce the following consequences.

(a) The endo-group property may not pass to fully invariant subgroups.

(b) Arbitrary direct sums of torsion endo-groups are endo-groups.

(c) Any group, containing a mixed or torsion-free endo-group as the direct summand is an endo-group.

\section{Three extreme classes}

Related to the class of groups considered in [7], one may consider the class of groups $G$ in which the only endo-images are 0 and $G$ (endo-image simple, for short). Obviously, the simple groups, i.e. $\mathbb{Z}(p)$ for any prime $p$, belong to both classes.

Proposition 6. A group $G$ is endo-image simple iff $G \cong \mathbb{Z}\left(p^{\infty}\right)$ or $G \cong \mathbb{Z}(p)$ for some prime $p$ or $G \cong \mathbb{Q}$. 
Proof. A group $G$ is endo-image simple iff all non-zero endomorphisms are surjective. From this the claim follows.

It is also natural to consider pure subgroups in a similar study and to determine the groups such that no pure subgroup is an endo-image (pure-image simple, for short), or, the groups all of whose pure subgroups are endo-images. This is done in the remainder of this section.

As for pure-image simple, we start by observing that since direct summands are pure, such a group must be indecomposable, so cocyclic or torsion-free.

Since a group $G \neq 0$ is pure-simple iff $r(G)=1$, all cocyclic groups are endopure simple and these are the only torsion groups with this property. The same argument works for all rational groups (not only $\mathbb{Q}$ ) since these are pure-simple.

Since mixed groups are not indecomposable, it remains to determine the pureimage simple torsion-free groups which are indecomposable and of rank at least 2.

First observe that since pure-image simple groups are indecomposable, these cannot contain non-trivial isomorphic pure subgroups. Since in [2] the groups with isomorphic non-trivial pure subgroups were studied, we infer that a pure-image simple group is divisible or reduced. As is well known, any divisible pure-image simple group is isomorphic to $\mathbb{Q}$ or $\mathbb{Z}\left(p^{\infty}\right)$ for some prime $p$. Any reduced pureimage simple group is either torsion, i.e. cyclic $p$-group for some prime $p$, or else an indecomposable torsion-free group.

This enables us to describe pure-image simple groups for a large class of groups: the cotorsion groups (i.e. groups whose extensions by torsion-free groups split). Recall from [5, (54.4)] that a torsion group is cotorsion iff it is a direct sum of a divisible group and a bounded group, and from [5, (54.5)] that a torsion-free group is cotorsion iff it is algebraically compact (i.e. $G$ is algebraically compact if $G$ is a direct summand in every group $H$ that contains $G$ as pure subgroup). By a theorem of Kaplansky [5, (40.4)], every non-zero reduced algebraically compact group contains a direct summand isomorphic to $J_{p}$ (the group of p-adic integers) or $\mathbb{Z}\left(p^{k}\right)$ for some positive integer $k$ and some prime $p$. It follows that the directly indecomposable algebraically compact groups are $J_{p}, \mathbb{Q}$, and the subgroups of $\mathbb{Z}\left(p^{\infty}\right)$. Therefore:

Proposition 7. A non-zero cotorsion group $G$ is pure-image simple iff $G$ is a divisible pure-image simple group or a cyclic p-group or the group of $p$-adic integer numbers for some prime $p$.

Before proceeding, we recall the following definitions (see [6, Section 92]). Let $G$ and $H$ be torsion-free groups of finite rank such that $G$ is contained in the divisible hull of $H$. Then $G$ is quasi-contained in $H$ if $n G \leq H$ for some positive integer $n$, and quasi-equal to $H$ if also $H$ is quasi-contained in $G$. A group $G$ 
is a quasi-direct sum of subgroups $K_{1}, \ldots, K_{m}$ of its divisible hull if $G$ is quasiequal to $K_{1} \oplus \cdots \oplus K_{m}$.

A group having only trivial quasi-direct decompositions is called strongly indecomposable.

In order to determine the remaining pure-image simple torsion-free groups, we first need the following:

Lemma 8. (1) If $G$ is a pure-image simple torsion-free group, then the type of any of its torsion-free factor-groups of rank 1 cannot be the type of any non-zero element of group $G$.

(2) Any pure-image simple torsion-free group $G$ of rank $\leq 3$ is strongly indecomposable.

(3) Any homogeneous torsion-free group $G$ of rank $\leq 3$ is pure-image simple iff it is strongly indecomposable.

Proof. (1) Indeed, if a factor-group $G / A$ is torsion-free of rank 1 and $\mathbf{t}(G / A)=$ $\mathbf{t}(a)$ for some $0 \neq a \in G$, then the pure subgroup $\langle a\rangle_{*}$ is an endo-image.

(2) By way of contradiction, assume the group $G$ is quasi-decomposable. Then $n G \leq A \oplus B \leq G$ for some positive integer $n$, where $A$ is a pure subgroup of rank 1 and $B$ is a pure subgroup of rank 1 or 2 and so $G / B \cong A$ (actually $G / B$ is quasi-isomorphic to $A$, but since the rank of $A$ is 1 , quasi-isomorphisms are isomorphisms). Hence $A$ is an endo-image, a contradiction.

(3) If the factor-group $G /(A \oplus B)$ is not bounded for all pure subgroups $A$ and $B$ of rank 1 and $\leq 2$, respectively, then by the homogeneous hypothesis, both types $\mathbf{t}(G / B), \mathbf{t}(G / A)>\mathbf{t}(G)$, i.e. any non-zero pure subgroup rank 1 or rank 2 of $G$ is not an endo-image. That the condition is necessary follows from (2).

Notice that the converses of (2) and (3) both fail.

Example 9. (1) Strongly indecomposable torsion-free groups $G$ of rank 2 which are not pure-image simple do exist.

(2) Strongly indecomposable not homogeneous pure-image simple torsion-free groups $G$ of rank 2 do exist.

Proof. (1) Let $A$ and $B$ be torsion-free groups of rank 1 such that $\mathbf{t}(A)=\mathbf{t}(B)$ and $p A \neq A, p B \neq B$ for some prime number $p$. Consider a subgroup of the divisible hull of the group $A \oplus B$, namely $G=\left\langle A \oplus B, p^{-\infty}(a+b)\right\rangle$, where $0 \neq a \in A$, $0 \neq b \in B$ are fixed elements and $p^{-\infty}(a+b)=\left\{p^{-1}(a+b), p^{-2}(a+b), \ldots\right\}$. Note that $A$ and $B$ are pure subgroups of $G$. Indeed, (say) for $A$, suppose that 
$n x=a^{\prime} \in A$ for some positive integer $n$ and $x \in G \backslash(A \oplus B)$. Then

$$
\begin{aligned}
& x=s_{1} x_{1}+\cdots+s_{m} x_{m}, \\
& \text { where } x_{1}=p^{-k_{1}}(a+b), \ldots, x_{m}=p^{-k_{m}}(a+b) \text {. If } k=\max \left\{k_{1}, \ldots, k_{m}\right\} \text {, then } \\
& \qquad \begin{aligned}
n p^{k} x & =n s_{1} p^{k} x_{1}+\cdots+n s_{n} p^{k} x_{m} \\
& =n\left(s_{1} p^{k-k_{1}}+\cdots+s_{m} p^{k-k_{m}}\right) a+n\left(s_{1} p^{k-k_{1}}+\cdots+s_{m} p^{k-k_{m}}\right) b \\
& =p^{k} a^{\prime} .
\end{aligned}
\end{aligned}
$$

Since all elements in this equality belong to $A \oplus B$,

$$
n\left(s_{1} p^{k-k_{1}}+\cdots+s_{m} p^{k-k_{m}}\right) b=0
$$

and so $s_{1} p^{k-k_{1}}+\cdots+s_{m} p^{k-k_{m}}=0$. Hence $x=0$, since all considered groups are torsion-free. Finally, observe that $\mathbf{t}(G / B)=\mathbf{t}(a+b)$ and so the pure subgroup $\langle a+b\rangle_{*}$ is an endo-image.

(2) Let $A, B$ be torsion-free groups of rank 1 such that $p_{1} A=A, p_{2} B=B$ and $p_{2} A \neq A, p_{1} B \neq B$ for some prime numbers $p_{1} \neq p_{2}$. Now consider a subgroup of the divisible hull of the group $A \oplus B$, namely $G=\left\langle A \oplus B, p^{-\infty}(a+b)\right\rangle$, where $0 \neq a \in A, 0 \neq b \in B$ and $p$ is a some prime number with $p A \neq A, p B \neq B$.

Let $X$ be a pure subgroup of rank 1 of the group $G$. If $A \subseteq X$, then $G / X$ is $p$-divisible and $p_{2}$-divisible. But the group $G$ has no such non-zero elements. Similarly if $B \subseteq X$. If $X \neq A, B$ and $0 \neq x \in X$, then $n x=s a+t b$ for some $n, s, t \in \mathbb{Z}$. Then $s a+X=-t b+X$ so $G / X$ is $p_{1}$-divisible and $p_{2}$-divisible. But the group $G$ has no such non-zero elements. Consequently, the group $G$ is pure-image simple.

Moreover, we can provide the following:

Example 10. Pure-image simple torsion-free groups of rank $n$ exist for any positive integer $n$. For any infinite cardinal number $\mathfrak{m}$, less than the first strongly inaccessible cardinal number, pure-image simple torsion-free groups of power $\mathfrak{m}$ do exist.

Proof. For the first example, take the reduced cohesive group (a torsion-free group with only divisible factor groups modulo non-zero pure subgroups; see [6] or [10]) $G$ of rank $n$. Every $f \in E$ is a monomorphism, so $G$ has no isomorphic non-trivial pure subgroups.

For the second example, take any group $A$ of cardinality $\mathfrak{m}$ from a rigid system (such groups exist according to [6, Theorem 89.2]). Since any endomorphism of $A$ is a multiplication with a rational number, $A$ has no non-trivial pure endoimages. 
Alternatively, for every $n$ there is a rank $n$ group with endomorphism ring isomorphic to $\mathbb{Z}$. For infinite rank there is a whole class of torsion-free groups with endomorphism ring isomorphic to $\mathbb{Z}$. Any of these groups are also examples of pure-image simple groups.

Next recall that a group $A$ is called sp-group, if it is a reduced mixed group with infinitely many non-zero $p$-components $A_{p}$, such that the natural embedding $\bigoplus_{p} A_{p} \rightarrow A$ can be extended to a pure embedding $A \rightarrow \prod_{p} A_{p}$.

In [1], the following criterion for a group to be a sp-group was established.

Theorem 11. The following conditions are equivalent for a reduced mixed group $A$ with infinitely many non-zero p-components $A_{p}$ :

(1) $A$ is a sp-group, i.e., the pure embeddings $\bigoplus_{p} A_{p} \subset A \subseteq \prod_{p} A_{p}$ hold.

(2) Both embeddings $\bigoplus_{p} A_{p} \subset A \subseteq \prod_{p} A_{p}$ hold and $A /\left(\bigoplus_{p} A_{p}\right)$ is a divisible torsion-free group.

(3) For each prime $p$, there is a group $B_{p}$ such that $A=A_{p} \oplus B_{p}$ with $p B_{p}=B_{p}$.

We can now characterize the third class of groups, the groups all of whose endoimages are pure subgroups.

Proposition 12. In a group $G$ all endo-images are pure subgroups iff $G$ is one of the following groups:

(1) $G$ is a divisible group,

(2) $G$ is a torsion group and every p-component is elementary or divisible,

(3) any p-component of $G$ is elementary or divisible and the reduced part of $G$ is a sp-group.

Proof. In order to show that the conditions are necessary, we use multiplication by a prime number $p$ as an endomorphism of $G$. Since $p G$ is (by hypothesis) pure in $G$, we get $p G=G \cap p G=p(p G)$, i.e. $p G$ is $p$-divisible. So if $p G_{p} \neq 0$, then the $p$-component $G_{p}$ is divisible. If $p G_{p}=0$, then $G=G_{p} \oplus B_{p}$ with $p B_{p}=B_{p}$ and this completes the proof by Theorem 11 .

As for sufficiency, let $H=f(G), f \in E$, be an endo-image of $G$. Since any $p$-component $G_{p}$ is divisible or elementary and $G=G_{p} \oplus B_{p}$, where $p B_{p}=B_{p}$, any $p$-component $H_{p}$ is divisible or elementary, and $H=H_{p} \oplus C_{p}$ for some subgroup $C_{p} \leq H$. If $H_{p}$ is divisible, then $G_{p}$ is divisible and $p G=G$ implies $p H=H$. But if $H_{p}$ is elementary, then so is $G_{p}$ and $p B_{p}=B_{p}$ implies $f\left(G_{p}\right) \cap$ $f\left(B_{p}\right)=0$. Hence $H=f(G)=f\left(G_{p}\right) \oplus f\left(B_{p}\right)$ and so $C_{p} \cong f\left(B_{p}\right)$. In particular, $C_{p}$ is $p$-divisible. Therefore $H_{p}$ is $p$-pure in $G$ and so is $C_{p}$ since it is $p$-divisible. Finally, $H$ is pure in $G$. 


\section{Ker-gen groups}

The notion studied in this section was suggested by a similar one given in [12] for $R$-modules.

We say that an $R$-module $M$ generates its kernels (ker-gen for short) if for every $f \in \operatorname{End}_{R}(M), \operatorname{ker}(f)$ is $M$-generated. This notion was introduced in [12] in order to compare morphic modules with modules whose endomorphism ring is left morphic. An $R$-module $M$ is called morphic if $M / f(M) \cong \operatorname{ker}(f)$ for every endomorphism $f \in \operatorname{End}_{R}(M)$, and a ring $R$ is called left morphic if ${ }_{R} R$ is morphic.

In the sequel we discuss these notions for $\mathbb{Z}$-modules, that is, for Abelian groups.

Both morphic groups and endo-groups are included in the class of ker-gen groups. However, these two subclasses are not related: $\mathbb{Q}$ is morphic but no endogroup, $\mathbb{Z}$ is endo-group but not morphic.

From [3] we already have a list of ker-gen groups:

(i) A torsion-free group is morphic only if it is divisible.

(ii) A divisible group is morphic only if it is torsion-free. This occurs iff

$$
G \cong \mathbb{Q} \oplus \mathbb{Q} \oplus \cdots \oplus \mathbb{Q},
$$

i.e. a finite direct sum of $\mathbb{Q}$.

Therefore, the morphic torsion groups are reduced.

(iii) A torsion group is morphic iff all its primary components are morphic.

(iv) $\mathrm{A}$ (reduced) $p$-group $G$ is morphic iff it is finite and homogeneous.

(v) The splitting morphic mixed groups are exactly the groups

$$
G=T(G) \oplus D(G)=\bigoplus_{p}\left(\mathbb{Z}\left(p^{k_{p}}\right)^{n_{p}} \oplus \mathbb{Q}^{k}\right.
$$

with non-negative integers $k_{p}, n_{p}$, and $k$.

Another known situation when a module $M$ generates its kernels is when $\operatorname{ker} f$ is a direct summand of $M$ for every $f \in \operatorname{End}_{R}(M)$. We say that $M$ is kernel-direct in this case. As is well known, this happens if $\operatorname{End}(M)$ is regular. Then we have another list of ker-gen groups (see [6, Section 112.7]):

(a) If $G$ is a direct sum of a torsion-free divisible group and an elementary group, then it is kernel-direct and so ker-gen.

(b) Elementary groups are kernel-direct and so ker-gen.

For the main result of this section recall that for a set $\Pi$ of prime numbers, $\mathbb{Q}_{\Pi}$ denotes the group (ring) of all rational numbers with denominator coprime to all $p \in \Pi$. 
Theorem 13. (1) A torsion group $G$ is ker-gen iff every unreduced p-component $G_{p}$ has an unbounded reduced part.

(2) A divisible group is ker-gen iff it is torsion-free.

(3) Let $G=R \oplus D$, where $D=\bigoplus_{p \in \Pi} D_{p} \oplus D_{0}$ is the divisible part of $G, R$ is reduced, $D_{0}$ is a torsion-free part of $D$ and $\Pi$ is the set of primes with $D_{p} \neq 0$. Then $G$ is ker-gen iff the following conditions hold:

(i) $R$ is ker-gen with $p^{n} R \neq p^{n-1} R$ for all $p \in \Pi$ and every positive integer $n$,

(ii) if $D_{0} \neq 0$ there is a subgroup $H$ of $R$ such that the factor-group $R / H$ is torsion-free of rank 1 and $\mathbf{t}(R / H) \leq \mathbf{t}(\mathbb{Q} \Pi)$,

(iii) the kernel of any homomorphism $R \rightarrow D$ is $R$-generated.

Proof. (1) Assume that $G_{p}$ has a direct summand isomorphic to $\mathbb{Z}\left(p^{\infty}\right)$. Since there are epimorphisms of $\mathbb{Z}\left(p^{\infty}\right)$ with kernels of arbitrary large order, it follows that $G_{p}$ has cyclic direct summands of arbitrary large order, i.e. the reduced part of $G_{p}$ is unbounded. Conversely, the statement follows since all subgroups of $G$ are cyclic direct summands of $G$.

(2) Obvious, since homomorphic images of divisible groups are divisible.

(3) If $G$ is ker-gen, clearly $R$ is ker-gen and the kernel of any homomorphism $R \rightarrow D$ is $R$-generated. Since $\mathbb{Z}\left(p^{\infty}\right)$ has endomorphisms with kernels of arbitrary large order, it follows that $p^{n} R \neq p^{n-1} R$ for all $p \in \Pi$ and positive integers $n$. Finally, since $\mathbb{Q} / \mathbb{Q}_{\Pi} \cong \bigoplus_{\Pi} \mathbb{Z}\left(p^{\infty}\right)$, we have $\operatorname{Hom}\left(R, \mathbb{Q}_{\Pi}\right) \neq 0$ and so such a subgroup $H$ exists.

Conversely, first notice that every endomorphism of the group $G$ is determined by three homomorphisms (in an upper triangular matrix form): an endomorphism of $R$, a homomorphism $R \rightarrow D$ and an endomorphism of $D$. In view of conditions on the group $R$, it only remains to show that the kernel of every endomorphism of $D$ is $G$-generated. Every endomorphism of the group $D$ is also determined by three homomorphisms: an endomorphism of the group $D_{0}$, a homomorphism $D_{0} \rightarrow T(D)$ and an endomorphism of the group $T(D)$. The kernel of the endomorphism of $D_{0}$ is a direct summand of $D_{0}$. Let $D_{0} / F$ be isomorphic to some subgroup of $T(D)$. Then $p F=F$ for every prime $p$ not in $\Pi$ and so $F$ is a $\mathbb{Q}_{\Pi}$-module. Hence $F$ is generated by homomorphic images of the group $\mathbb{Q}_{\Pi}$. Finally, since $p^{n} R \neq p^{n-1} R$ for all $p \in \Pi$ and positive integers $n, R / p^{n} R$ contains the cyclic groups of order $p^{n}$, the whole group $T(D)$ is $R$-generated.

Kernels of endomorphisms of any reduced torsion-free group are closed pure subgroups. We mention that in [4] groups in which every closed pure subgroup is a direct summand were studied. Such groups are clearly ker-gen. 
Lemma 14. Let $G$ be a separable torsion-free group and let $\Omega$ be the set of types of rank 1 direct summands of $G$.

(i) Every pure subgroup of $G$ is $G$-generated iff for any $\mathbf{t}_{1}, \mathbf{t}_{2} \in \Omega$ there is $\boldsymbol{\tau} \in \Omega$ with $\boldsymbol{\tau} \leq \mathbf{t}_{1}, \mathbf{t}_{2}$.

(ii) Assume that for any $\mathbf{t}_{1}, \mathbf{t}_{2} \in \Omega$ there is $\boldsymbol{\tau}^{\prime} \in \Omega$ with $\boldsymbol{\tau}^{\prime} \geq \mathbf{t}_{1}, \mathbf{t}_{2}$. Then $G$ is ker-gen iff or any $\mathbf{t}_{1}, \mathbf{t}_{2} \in \Omega$ there is $\boldsymbol{\tau} \in \Omega$ with $\boldsymbol{\tau} \leq \mathbf{t}_{1}, \mathbf{t}_{2}$.

Proof. (i) Let $G=G_{1} \oplus A=G_{2} \oplus B$, where $r\left(G_{1}\right)=r\left(G_{2}\right)=1$, and $\mathbf{t}_{1}=\mathbf{t}\left(G_{1}\right)$ and $\mathbf{t}_{2}=\mathbf{t}\left(G_{2}\right)$ are incomparable. Then $G_{2} \subseteq G\left(\mathbf{t}_{2}\right)=\left\{g \in G: \mathbf{t}(g) \geq \mathbf{t}_{2}\right\}$ and $G\left(\mathbf{t}_{2}\right)=\left(G\left(\mathbf{t}_{2}\right) \cap G_{1}\right) \oplus\left(A \cap G\left(\mathbf{t}_{2}\right)\right)$. Since $G\left(\mathbf{t}_{2}\right) \cap G_{1}=0, G\left(\mathbf{t}_{2}\right) \subseteq A$ follows and so $G_{1} \oplus G_{2}$ is a direct summand of $G$. If $0 \neq x \in G_{1}, 0 \neq y \in G_{2}$, then since $\langle x+y\rangle_{*}$ is $G$-generated, $\boldsymbol{\tau} \leq \mathbf{t}(x+y)<\mathbf{t}_{1}, \mathbf{t}_{2}$ for some $\tau \in \Omega$.

Conversely, let $H$ be a pure subgroup of $G$ and $x \in H$. Since $G$ is separable, $\mathbf{t}(x)=\mathbf{t}_{1} \cap \cdots \cap \mathbf{t}_{n}$ for some $\mathbf{t}_{1}, \ldots, \mathbf{t}_{n} \in \Omega$. From the hypothesis it follows that there exists $\boldsymbol{\tau} \in \Omega$ with $\boldsymbol{\tau} \leq \mathbf{t}_{1} \cap \cdots \cap \mathbf{t}_{n}$. If $A$ is a direct summand of rank 1 and $\mathbf{t}(A)=\boldsymbol{\tau}$, then $S_{A}\left(\langle x\rangle_{*}\right)=\langle x\rangle_{*}$. Each homomorphism $A \rightarrow\langle x\rangle_{*}$ lifts to an endomorphism of the group $G$, mapping any complement of $A$ into 0 . Consequently, $H$ is $G$-generated.

(ii) Let $0 \neq x \in G_{1}, 0 \neq y \in G_{2}$, where $G_{1} \oplus G_{2}$ is a direct summand of $G$ and $\mathbf{t}\left(G_{1}\right), \mathbf{t}\left(G_{2}\right)$ are incomparable (see (i)). Take $F=\left(G_{1} \oplus G_{2}\right) /\langle x+y\rangle_{*}$, so that $r(F)=1$. Since $\mathbf{t}\left(G_{3}\right)>\mathbf{t}_{1}, \mathbf{t}_{2}$ for some direct summand $G_{3}$ of rank 1 , there is a homomorphism $g: F \rightarrow G_{3}$ and we have $G=G_{1} \oplus G_{2} \oplus C \oplus G_{3}$. Define the endomorphism $f$ as follows: $f\left|\left(G_{1} \oplus G_{2}\right)=g, f\right| C=1_{C}$ and $f\left(G_{3}\right)=0$. Then $\operatorname{ker}(f)=\langle x+y\rangle_{*} \oplus G_{3}$. Since $\operatorname{ker}(f)$ is $G$-generated, $\tau \leq$ $\mathbf{t}(x+y)<\mathbf{t}_{1}, \mathbf{t}_{2}$ for some $\boldsymbol{\tau} \in \Omega$. We are done now using (i).

Proposition 15. (1) If $G=G_{1} \oplus G_{2}$, where $G_{1}$ and $G_{2}$ are torsion-free groups of rank 1 , then $G$ is a ker-gen group. If the types $\mathbf{t}\left(G_{1}\right)$ and $\mathbf{t}\left(G_{2}\right)$ are incomparable, then $G$ has pure subgroups which are not $G$-generated.

(2) If $G=G_{1} \oplus G_{2} \oplus \mathbb{Q}$, where $G_{1}$ and $G_{2}$ are torsion-free groups of rank 1 of incomparable types, then $G$ is not ker-gen.

Proof. (1) If $0 \neq H \neq G$ is a kernel and $H \neq G_{1}, G_{2}$, then $H=\langle x+y\rangle_{*}$ for some $x \in G_{1}$ and $y \in G_{2}$. Since $G / H$ is isomorphic to some subgroup of $G$, $\mathbf{t}(G / H) \leq \mathbf{t}\left(G_{1}\right)$ or $\mathbf{t}(G / H) \leq \mathbf{t}\left(G_{2}\right)$. On the other hand $\mathbf{t}(G / H) \geq \mathbf{t}\left(G_{1}\right), \mathbf{t}\left(G_{2}\right)$, whence $\mathbf{t}\left(G_{1}\right) \leq \mathbf{t}\left(G_{2}\right)$ or $\mathbf{t}\left(G_{2}\right) \leq \mathbf{t}\left(G_{1}\right)$. But then we have $\mathbf{t}\left(G_{1}\right)=\mathbf{t}(x+y)$ or $\mathbf{t}\left(G_{2}\right)=\mathbf{t}(x+y)$ so $G_{1}$ or $G_{2}$ generates $H$. Now if the types $\mathbf{t}\left(G_{1}\right)$ and $\mathbf{t}\left(G_{2}\right)$ are incomparable then $\mathbf{t}(x+y)<\mathbf{t}\left(G_{1}\right), \mathbf{t}\left(G_{2}\right)$, so $H$ is not $G$-generated.

(2) Follows from the previous lemma. 
Since the structure of ker-gen groups was clarified above, it follows that the ker-gen property does not pass to (fully invariant) summands, as the property of being morphic does. As an example, let $T$ be an unbounded $p$-group. Then by Proposition 5, $T \oplus \mathbb{Z}\left(p^{\infty}\right)$ is an endo-group. However if $T_{1}$ is a bounded direct summand of $T$ then the group $T_{1} \oplus \mathbb{Z}\left(p^{\infty}\right)$ is not ker-gen.

\section{Duality}

In [8] a property (denoted Q) which is dual to the property $\mathrm{P}$, recalled in the Introduction, was studied.

Notice that $\mathrm{P}$ (every subgroup is an endo-image) is equivalent to the property that every subgroup is isomorphic to some factor group.

A group $G$ has property $Q$ if every homomorphic image of $G$ can be (isomorphically) embedded in $G$. In other words, every factor group of $G$ is isomorphic to some subgroup of $G$.

The following results were proved.

Theorem. An abelian p-group $G$ has property $Q$ iff it contains a direct summand of the form $\bigoplus_{\mathfrak{m}} \mathbb{Z}\left(p^{\infty}\right)$, where $\mathfrak{m}=\min _{n} \operatorname{rank}\left(p^{n} G\right)$ is the final rank of $G$.

Corollary. A reduced abelian p-group has property $Q$ iff it is bounded.

Theorem. An abelian group $G$ of infinite torsion free rank $r$ has property $Q$ iff

(i) $r \leq \mathfrak{p}_{i}$ holds for the final rank $\mathfrak{p}_{i}$ of the $p_{i}$-component $G_{p_{i}}$ of the torsion subgroup $T(G)$, for each prime $p_{i}$,

(ii) $G$ contains a direct summand of the form $\bigoplus_{r} \mathbb{Q} \oplus \bigoplus_{i} \bigoplus_{\mathfrak{p}_{i}} \mathbb{Z}\left(p_{i}^{\infty}\right)$.

Theorem. A group $G$ of finite torsion-free rank $r$ has property $Q$ iff

$$
G=F \oplus T=F \oplus \bigoplus_{i=1}^{\infty} T_{i},
$$

where

(i) every $T_{i}$ is a $p_{i}$-group of infinite final rank $\mathfrak{p}_{i}$,

(ii) every $T_{i}$ has property $Q$,

(iii) $F$ is a torsion free group of rank $r$,

(iv) $F=R\left(\sigma_{1}\right) \oplus \cdots \oplus R\left(\sigma_{r}\right)$, where $R\left(\sigma_{i}\right)$ are rational groups of type $\sigma_{i}$ satisfying $\sigma_{1} \geq \cdots \geq \sigma_{r}$. 
We first recall a dual construction to the $A$-socle, namely, the $A$-radical (or co-trace) of $B$ : for two groups $A$ and $B$,

$$
K_{B}(A):=\bigcap_{\alpha \in \operatorname{Hom}(A, B)} \operatorname{ker} \alpha,
$$

which is also a fully invariant subgroup of $A$. If $K_{B}(A)=0$, we say that $A$ is $B$-cogenerated.

Let $A, B_{i}(i \in I)$ be groups and let $f_{i}: A \rightarrow B_{i}(i \in I)$ be homomorphisms. If $f: A \rightarrow \prod_{i \in I} B_{i}$ is the factorization homomorphism, it is well known that $\operatorname{ker}(f)=\bigcap_{i \in I} \operatorname{ker}\left(f_{i}\right)$. Hence $K_{B}(A)=0$ (i.e. $A$ is $B$-cogenerated) iff $A$ embeds in a power of $B$ (i.e. a direct product of copies of $B$ ).

A group $G$ has the property Q if for every subgroup $H, G / H$ embeds in $G$, that is, iff there is a homomorphism $f: G / H \rightarrow G$ with $\operatorname{ker}(f)=0$ (i.e. monic).

The generalization we deal with in this section is the following: a factor group $G / H$ is $G$-cogenerated if

$$
K_{G}(G / H):=\bigcap_{f \in \operatorname{Hom}(G / H, G)} \operatorname{ker} f=0 .
$$

As seen above, $G / H$ is $G$-cogenerated iff $G / H$ embeds in a power of $G$ iff there is a monomorphism from $G / H$ to a direct product of copies of $G$.

Dual to endo-groups (determined in Section 2), a group $G$ will be called co-endo-group if all its factor groups are $G$-cogenerated.

In the sequel we determine the co-endo-groups.

Since any torsion-free group has torsion homomorphic images, it follows that any co-endo-group is a torsion or a mixed group.

Proposition 16. A torsion group $G$ is a co-endo-group iff every p-component $G_{p}$ is bounded or not reduced.

Proof. If $G_{p}$ is unbounded, then $G_{p} / H \cong \mathbb{Z}\left(p^{\infty}\right)$ for some subgroup $H$. Since a non-zero homomorphism $\mathbb{Z}\left(p^{\infty}\right) \rightarrow G_{p}$ does exist, it follows that $G_{p}$ is not reduced. The converse is obvious.

Theorem 17. A mixed group $G$ is a co-endo-group iff the group $G$ has subgroups isomorphic to $\mathbb{Z}\left(p^{\infty}\right)$, for every prime number $p$.

Proof. If the factor group $G / T(G)$ is not $p$-divisible, then any group of order $p^{n}$ is a homomorphic image and so every $p$-component $G_{p}$ is unbounded. Therefore 
$G_{p} / H \cong \mathbb{Z}\left(p^{\infty}\right)$ for some subgroup $H$, whence $G$ has a direct summand isomorphic to $\mathbb{Z}\left(p^{\infty}\right)$. In the remaining case, if $G / T(G)$ is $p$-divisible, again the group $G$ has a direct summand isomorphic to $\mathbb{Z}\left(p^{\infty}\right)$.

The converse follows from the fact that $G$ contains a subgroup isomorphic to $\mathbb{Q} / \mathbb{Z} \cong \bigoplus_{p} \mathbb{Z}\left(p^{\infty}\right)$, which is a coimage group.

\section{Open problems}

As we did in the Section 3 for pure subgroups, we may consider fully invariant subgroups instead. Since sums of fully invariant subgroups are fully invariant, the groups $G$ in which the G-generated subgroups are fully invariant are precisely the groups in which endomorphic images are fully invariant. These groups were studied in [11].

The converse problem could be of interest:

Problem 1. Describe the groups $G$ in which the fully invariant subgroups are $G$-generated.

A continuation for Section 4 could be:

Problem 2. Describe the (torsion-free) vector ker-gen groups.

Homogeneous torsion-free completely decomposable groups of finite rank and reduced algebraically compact groups $G$ have the following property: the pure subgroups which are endomorphic images are the direct summands of $G$. Therefore we may add:

Problem 3. Describe the groups $G$ in which all pure subgroups which are endomorphic images are direct summands of $G$.

Related to the classes described in Section 3 we also state:

Problem 4. Describe the groups in which every pure subgroup is an endomorphic image (or else, is a kernel of endomorphism).

Recall that a pure subgroup of a torsion group $G$, which is a direct sum of cyclic groups, is an endomorphic image of $G$ ([5, Section 36.2]).

Acknowledgments. Thanks are due to the referee for useful suggestions which improved the presentation of our paper. 


\section{Bibliography}

[1] U. F. Albrecht, H. P. Goeters and W. Wickless, The flat dimension of mixed abelian groups as E-modules, Rocky Mountain J. Math. 25 (1995), 569-590.

[2] R. A. Beaumont and R. S. Pierce, Isomorphic direct summands of abelian groups, Math. Ann. 153 (1964), no. 1, 21-37.

[3] G. Călugăreanu, Morphic Abelian groups, J. Algebra Appl. 9 (2010), no. 2, 185-193.

[4] A. R. Chekhlov, Abelian torsion-free CS-groups, Sov. Math. 34 (1990), no. 3, 103-106.

[5] L. Fuchs, Infinite Abelian Groups. Vol. 1, Academic Press, New York, 1970.

[6] L. Fuchs, Infinite Abelian Groups. Vol. 2, Academic Press, New York, 1973.

[7] L. Fuchs, A. Kertesz and T. Szele, On abelian groups whose subgroups are endomorphic images, Acta Sci. Math. (Szeged) 16 (1955), 77-88.

[8] L. Fuchs, A. Kertesz and T. Szele, On abelian groups in which every homomorphic image can be imbedded, Acta Sci. Math. Hung. 7 (1956), 467-475.

[9] B. Huisgen-Zimmermann, Endomorphism rings of self-generators, Pacific J. Math. 61 (1975), no. 2, 587-602.

[10] P. A. Krylov, A. V. Mikhalev and A. A. Tuganbaev, Endomorphism Rings of Abelian Groups, Kluwer, Dordrecht, 2003.

[11] D. A. Lawver, Abelian groups in which endomorphic images are fully invariant, J. Algebra 29 (1974), 232-245.

[12] K. Nicholson, A survey of morphic modules and rings, in: Advances in Ring Theory (Nanjing 2004), World Scientific, Hackensack (2005), 167-180.

Received March 23, 2018; revised March 31, 2018.

\section{Author information}

Grigore Călugăreanu, Department of Mathematics,

Babeş-Bolyai University, Cluj-Napoca, Romania.

E-mail: calu@math.ubbcluj.ro

Andrey R. Chekhlov, Faculty of Mechanics and Mathematics,

Tomsk State University, Tomsk, Russia.

E-mail: cheklov@math.tsu.ru

Piotr A. Krylov, Faculty of Mechanics and Mathematics,

Tomsk State University, Tomsk, Russia.

E-mail: krylov@math.tsu.ru 